\title{
An shRNA barcode screen provides insight into cancer cell vulnerability to MDM2 inhibitors
}

\author{
Thijn R Brummelkamp ${ }^{1,3,4}$, Armida WM Fabius ${ }^{1,4}$, Jasper Mullenders ${ }^{1}$, Mandy Madiredjo ${ }^{1}$, Arno Velds ${ }^{1,2}$, \\ Ron M Kerkhoven ${ }^{1,2}$, René Bernards ${ }^{1} \&$ Roderick L Beijersbergen ${ }^{1}$
}

The identification of the cellular targets of small molecules with anticancer activity is crucial to their further development as drug candidates. Here, we present the application of a large-scale RNA interference-based short hairpin RNA (shRNA) barcode screen to gain insight in the mechanism of action of nutlin-3 (1). Nutlin-3 is a small-molecule inhibitor of MDM2, which can activate the 53 pathway. Nutlin-3 shows strong antitumor effects in mice, with surprisingly few side effects on normal tissues ${ }^{1}$. Aside from p53, we here identify 53BP1 as a critical mediator of nutlin-3-induced cytotoxicity. 53BP1 is part of a signaling network induced by DNA damage that is frequently activated in cancer but not in healthy tissues $^{2}$. Our results suggest that nutlin-3's tumor specificity may result from its ability to turn a cancer cell-specific property (activated DNA damage signaling ${ }^{3}$ ) into a weakness that can be exploited therapeutically.

The tumor-suppressor gene TP53 (also called p53 and encoding the protein p53) is the gene most frequently mutated in human cancer. However, approximately $50 \%$ of all human tumors retain normal p53 (ref. 4). Direct activation of $\mathrm{p} 53$ in these tumors could in principle be used as a means to eradicate tumor cells. The activity of p53 is tightly regulated. p53 is activated in response to a variety of stresses, such as DNA damage, nutrient deprivation or oncogene activation, resulting in the transcriptional activation of target genes involved in growth arrest and apoptosis ${ }^{5}$. To protect healthy cells from the deleterious effects of uncontrolled $\mathrm{p} 53$ activation, $\mathrm{p} 53$ is subject to a negative feedback loop activated by the protein product of one of its target genes, MDM2. The protein MDM2 binds to p53, inhibits transcriptional activation, causes nuclear export and acts as an $\mathrm{E} 3$ ligase to target $\mathrm{p} 53$ for proteasomal degradation ${ }^{6,7}$.

One potential approach for activating p53 in tumor cells is to disrupt the interaction between MDM2 and p53 with the small molecule nutlin-3 (refs. 1,8). Nutlin-3 binds to MDM2, thereby preventing the interaction with p53 and causing p53 to be stabilized. Nutlin-3 has strong antitumor effects in vivo but, notably, few toxic effects in normal mice $^{1}$. In theory, activation of p 53 could be at least as deleterious to normal cells as to tumor cells, because the former are not attenuated in their ability to undergo apoptosis and are more obedient to growthinhibitory signals than are cancer cells. However, tumor cells that have lost the p53 gene through mutation seem to be exquisitely sensitive to reintroduction of the wild-type gene ${ }^{9}$. This phenomenon has never been understood in detail, and has often been attributed to the presence of other oncogenic mutations or to a differential wiring of the p53 network in tumor cells as compared to normal cells ${ }^{9}$. We report here a loss-of-function genetic screen designed to identify important components of the p53 network mediating the cytotoxic effect of nutlin-3 in human tumor cells.

A variety of approaches can be used for the identification of genes essential for a drug-induced phenotype $\mathrm{e}^{9-16}$, including the recently developed RNAi barcode screening technology ${ }^{10-13}$. Barcode screens use DNA microarrays to follow the relative abundance, under particular conditions, of each shRNA vector in a large population of cells infected with an shRNA vector library (Fig. 1a). Using retroviral infection, we introduced a collection of 23,742 different pRETRO-SUPER vectors designed to target 7,914 human genes for suppression by RNA interference (RNAi) into MCF-7 cells (which have wild-type p53) ${ }^{10}$. The infected cells were split into two pools, one of which was left untreated and was used as a reference while the other was exposed to $4 \mu \mathrm{M}$ nutlin3. MCF-7 cells respond to this concentration of nutlin-3 predominantly by undergoing cell-cycle arrest rather than apoptosis (data not shown and Fig. 2c), possibly because the cells have a deficiency in caspase-3 (ref. 14). After $14 \mathrm{~d}$ of selection with nutlin-3, we recovered shRNA cassettes by PCR amplification and analyzed them by hybridization to DNA microarrays as described ${ }^{10,15}$. A small number of shRNA vectors were consistently enriched in the population treated with nutlin-3 (Fig. 1b). As expected, one of these vectors that was positively selected in the presence of nutlin-3 as compared to untreated control cells is directed against p53 which validates the shRNA barcode approach used here. Loss of $\mathrm{p} 53$ confers resistance to nutlin-3 by removing the effector of MDM2 (ref. 1). In addition, several of the shRNA vectors we identified target proteins with known or predicted functions in p53-mediated pathways (Fig. 1c). Two of these shRNA vectors target p53-binding protein-1 (53BP1), through independent sequences. 53BP1 has been implicated

\footnotetext{
${ }^{1}$ Division of Molecular Carcinogenesis and Center for Biomedical Genetics and ${ }^{2}$ Central Microarray Facility, The Netherlands Cancer Institute, Plesmanlaan 121,1066 CX Amsterdam, The Netherlands. ${ }^{3}$ Present address: Whitehead Institute for Biomedical Research, Nine Cambridge Center, Cambridge, Massachusetts 02142 , USA.

${ }^{4}$ These authors contributed equally to this work. Correspondence should be addressed to R.B. (r.bernards@nki.nl) or R.L.B. (r.beijersbergen@nki.nl).
} 
a
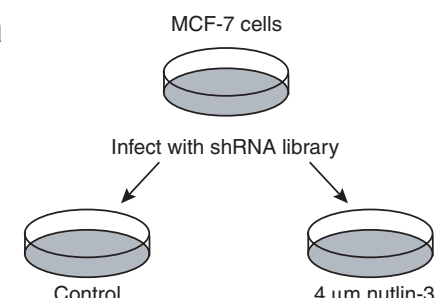

$4 \mu \mathrm{m}$ nutlin-3

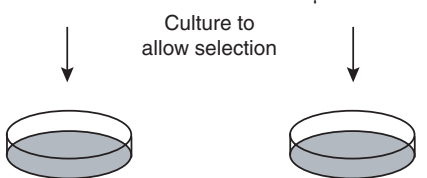

Isolate genomic DNA;

recover, amplify and

label shRNA cassettes

Array hybridization

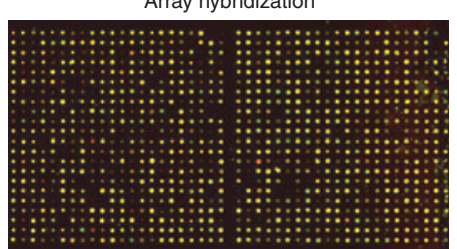

b

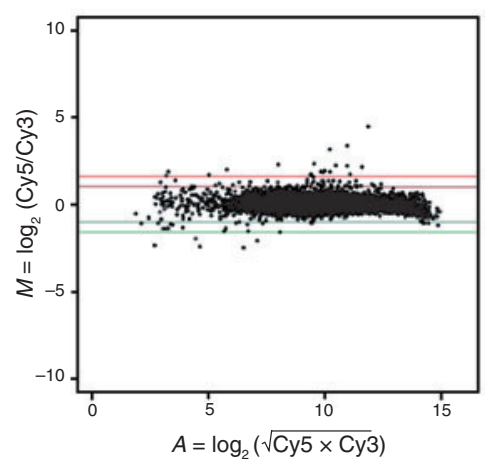

C

Description

Tumor protein p53

Tumor protein $\mathrm{p} 53$ binding protein

Tumor protein p53 binding protein 1

v-myc myelocytomatosis viral oncogene homolog

TGFB inducible early growth response

LIM homeobox protein 3

Potassium channel, subfamily K, member 9

RAB2, member RAS oncogene family

Immediate early response 3

Prostate differentiation factor

Glycine receptor, alpha 2

biquitin specific protease 28

Heterogeneous nuclear ribonucleoprotein $\mathrm{K}$
Figure 1 shRNA barcode screen identifies 53BP1 as a mediator of the antiproliferative effects of nutlin-3. (a) Schematic outline of the nutlin-3 barcode experiment in MCF-7 cells. Identical populations of MCF-7 cells infected with the NKI shRNA library were either left untreated or treated with $4 \mu \mathrm{M}$ nutlin-3. After $14 \mathrm{~d}$ the shRNA cassettes were recovered and labeled ${ }^{10,11}$. The labeled shRNA probes were hybridized to DNA microarrays containing the sequences complementary to the hairpin oligos. (b) Analysis of the relative abundance of shRNAs recovered from the nutlin-3 barcode experiment. Data are normalized and depicted as $M$, the ${ }^{2} \log$ (ratio Cy5/Cy3), versus $A\left({ }^{2} \log \right.$

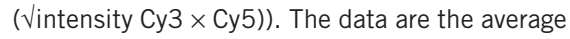
of two independent hybridization experiments performed in duplicate with reversed color. (c) List of genes targeted by the shRNA vectors from b that were significantly enriched $(P<0.01)$ in nutlin-3-treated cells. The vectors for TP53 and both vectors for 53BP1 have been validated for knockdown and rescue of nutlin-3-induced cell-cycle arrest. Column $M$ depicts the ${ }^{2}$ log ratio of the abundance of barcodes present in cells treated with nutlin-3 (Cy5) as compared to untreated cells (Cy3). in DNA damage sensing leading to p53 activation. In addition, we found an shRNA vector targeted against protein heterogeneous nuclear ribonucleoprotein $\mathrm{K}$ (hnRNPK), which has recently been identified as an MDM2 target and transcriptional coactivator of p53 in response to DNA damage ${ }^{16}$. The observation that depletion of hnRNPK abrogates the transcriptional induction of p53 target genes is consistent with an override of a nutlin-3-mediated cell-cycle arrest upon hnRNPK inactivation. We also identified three vectors targeting genes encoding known p53 transcriptional targets: BCL2-antagonist of cell death (BAD), placental bone morphogenic protein (PLAB) and immediate-early response 3 (IER3, also called IEX-1). Both BAD and IEX-1 have roles in the regulation of apoptosis. IEX-1 can block the PI3K/AKT pathway, resulting in increased sensitivity to apoptosis ${ }^{17}$. PLAB is also implicated as an important downstream mediator of DNA damage signaling ${ }^{18}$. Other shRNA vectors we identified in our screen target genes that are not implicated in the regulation of p53 or p53-dependent pathways. The presence of the genes encoding TGF $\beta$-inducible early growth response-1 (TIEG1, also called KLF10) and PLAB among the targets of the vectors identified suggests that the TGF $\beta$ pathway is involved in the cell-cycle arrest induced through the activation of the p53 pathway a
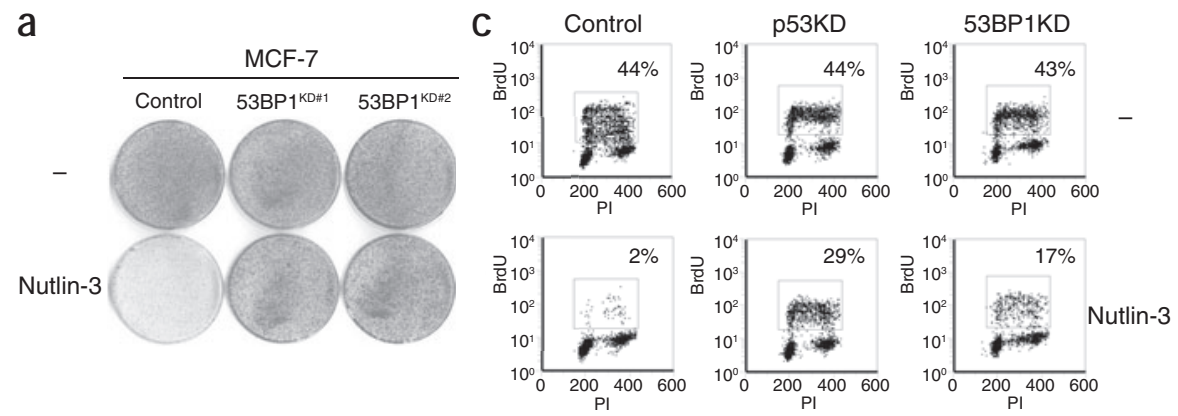

b

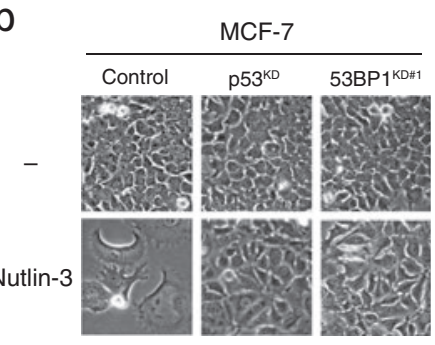

\section{d}
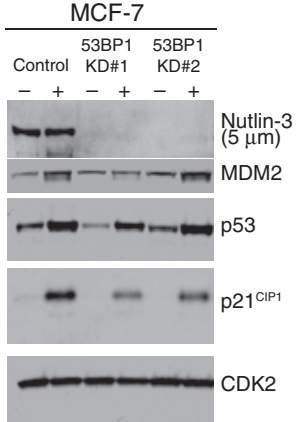

Figure 2 53BP1 modulates the cellular cytotoxicity induced by nutlin-3 in MCF-7 cells. (a) MCF-7 cells infected with shRNAs against GFP or two independent shRNAs directed against 53BP1 (knockdown vector is labeled $\mathrm{KD}$ ), untreated or treated with $4 \mu \mathrm{M}$ nutlin-3 for 14 days. Cell density is visualized by Coomassie staining of the cells. (b) MCF-7 cells and A549 cells infected with shRNAs directed against GFP, p53 or 53BP1, untreated or treated with nutlin-3. (c) Cell-cycle profile and BrdU incorporation of MCF-7 infected with shRNAs against GFP (control), p53 or 53BP1. Percentages of BrdUpositive cells are shown. (d) Western blot analysis of the MCF-7 cells expressing either shRNA against GFP (control) or one of the two shRNAs against 53BP1, 53BP1\#1 or 53BP1\#2. The cells were analyzed for the expression levels of 53BP1, the stabilization of $\mathrm{p} 53$ and the induction of $\mathrm{p} 21$ and MDM2. 
Figure 3 Intrinsic DNA damage signaling contributes to the cytotoxic effect of nutlin-3 in MCF-7 cells. (a) Immunolocalization of p53BP1 (above) and CHK2 (below) in human primary fibroblasts (left) and MCF-7 cells (right) before and after irradiation. (b) Western blot analysis of the stabilization of p53 and induction of p21 in MCF-7 cells treated with increasing concentrations nutlin-3 for $18 \mathrm{~h}$ in the absence or presence of $2.5 \mathrm{mM}$ caffeine. The cells were pretreated with $2.5 \mathrm{mM}$ caffeine for $12 \mathrm{~h}$. (c) Western blot analysis of the stabilization of p53 and the induction of p21 in normal BJ fibroblasts treated with increasing concentrations nutlin-3 without or with exposure to 2Gy irradiation.

by nutlin-3 treatment. Notably, TIEG1 expression is reduced in invasive breast cancer cells ${ }^{19}$. The shRNA barcode screen did not identify genes that enhance the cytotoxic effects of nutlin-3 in MCF-7 cells-that is, there were no shRNA vectors that were significantly depleted from the population as a result of nutlin-3 treatment. This may either be a consequence of the biological system or reflect a technical limitation of the current barcode protocol. In addition, we cannot exclude the possibility that some vectors are selected in this screen as a result of effects on cellular components other than the intended targets.

Our identification of two independent shRNA vectors targeted against $53 \mathrm{BP} 1$ in our screen greatly reduces the possibility that the observed effects are due to 'off-target' effects of the RNAi vectors. Moreover, this indicates that the observed phenotype is due to the reduced expression of the intended target. We therefore focused our subsequent validation on the effect of 53BP1 on nutlin-3 cytotoxicity. To validate the selection of shRNAs against 53BP1 in our barcode experiment, we performed a long-term growth assay with MCF-7 cells expressing the two different shRNA vectors against 53BP1. MCF-7 cells exposed to nutlin-3 undergo a proliferation arrest and acquire a flattened phenotype (Fig. 2a,b, left). In contrast, MCF-7 cells transduced with shRNA encoding retroviruses against p53 or 53BP1 and exposed to nutlin-3 show a cellular morphology resembling that of untreated MCF-7 cells. This effect is not unique to MCF-7 breast cancer cells, as inhibition of 53BP1 also alleviates the cytotoxic effects of nutlin-3 in A549 (human lung cancer) cells (Fig. 2b, right). We tested the effect of the inactivation of either p53 or 53BP1 on the cell-cycle progression of MCF-7 cells exposed to $4 \mu \mathrm{M}$ nutlin-3 for $48 \mathrm{~h}$. The percentage of MCF-7 cells in S-phase, as determined by BrdU incorporation, was reduced from $44 \%$ for control cells to $2 \%$ in cells exposed to nutlin-3, whereas knockdown of p53 or 53BP1 largely prevented this reduction, resulting in S-phase percentages of $29 \%$ and $17 \%$, respectively (Fig. 2c). To further validate the efficacy of the shRNA vectors against 53BP1, we tested their effects on the expression of 53BP1, the stabilization of $\mathrm{p} 53$ by nutlin-3, and the activation of a p53 target gene, CDKN1A (also called $p 21$ and encoding p $21^{\text {cip } 1}$ ). Both shRNA vectors targeting 53BP1 were active in inhibiting 53BP1 expression (Fig. 2d). The induction of $\mathrm{p} 53$ by nutlin-3 treatment is not altered in the presence or absence of 53BP1 expression. Notably, the shRNA-mediated knockdown of 53BP1 impairs the activation of the $\mathrm{p} 53$ target gene CDKN1A by nutlin-3. These results demonstrate that, beside $\mathrm{p} 53,53 \mathrm{BP} 1$ also has an essential role in mediating the cytotoxic effects of nutlin-3 in MCF-7 cells.

$53 \mathrm{BP} 1$ is a component of the ATM-CHK-53BP1 pathway that is activated by double-stranded DNA breaks or shortened telomeres, resulting in the induction of p53 (ref. 20). Notably, this ATM-CHK53BP1 DNA damage checkpoint-signaling pathway is often constitutively activated in human cancers and premalignant lesions, but not in normal, healthy tissues ${ }^{2,3,21}$. Based on the role of 53BP1 in mediating the antiproliferative effect of nutlin-3 identified here, we hypothesized that intrinsic DNA damage signaling in MCF-7 cells is required for nutlin-3 cytotoxicity. The presence of activated DNA damage signaling a

B

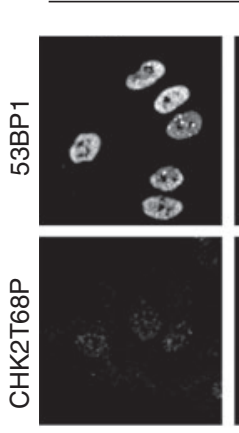

BJ
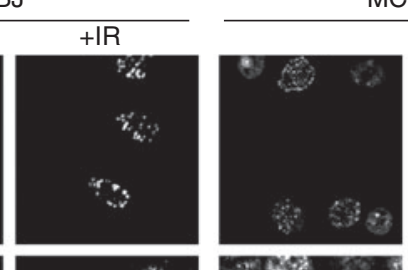

MCF-7
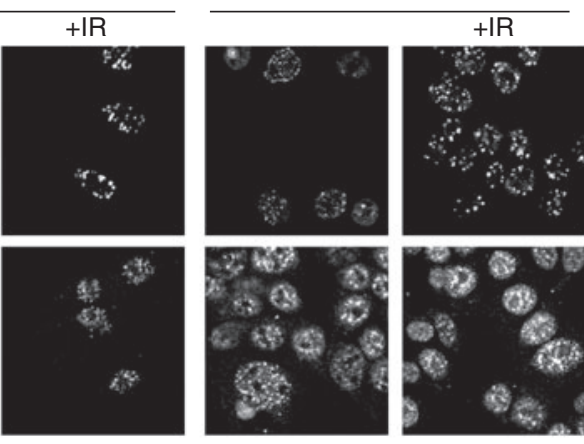

b

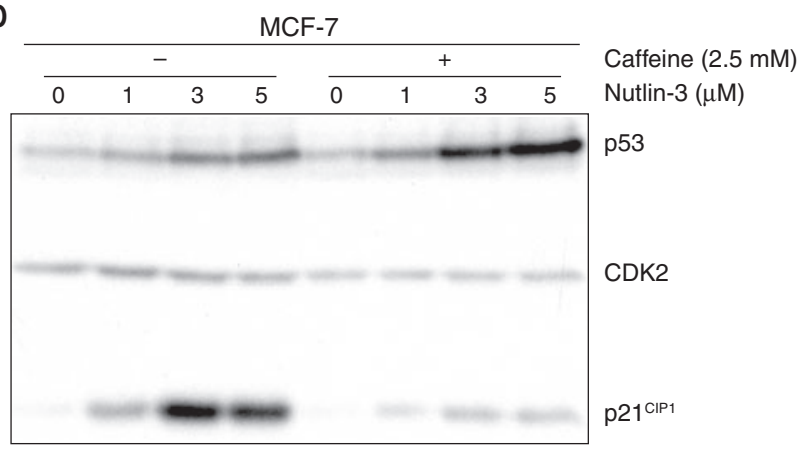

C

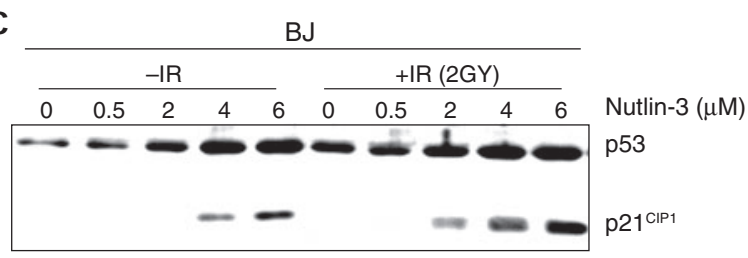

in MCF-7 cells was demonstrated by the detection of nuclear foci containing 53BP1, a characteristic of DNA damage-induced activation of 53BP1 (ref. 22). Indeed, a substantial fraction of MCF-7 cells show localization of 53BP1 in nuclear foci. Normal BJ fibroblasts have diffuse nuclear staining, although some 53BP1 foci are present (Fig. 3a, above). The presence of DNA damage foci in normal human fibroblasts in culture is in agreement with previous studies describing the presence of H2AX DNA damage foci and p53 activation in primary cells cultured in vitro ${ }^{23}$. MCF-7 cells also show strong nuclear staining with an antibody directed against phosphorylated CHK-2, even in the absence of ionizing radiation (Fig. 3a, below). In contrast, normal BJ fibroblasts show very little immunoreactivity, whereas BJ fibroblasts treated with ionizing radiation show strong nuclear staining (Fig. 3a, below). Together these data indicate that in MCF-7 cells, in the absence of ionizing irradiation, activation of DNA damage checkpoint signaling involving 53BP1 occurs, reflecting DNA damage signaling seen in many human tumors ${ }^{2}$.

The presence of activated DNA damage checkpoints in MCF7 cells suggests that DNA damage-induced 'stress signals' could be important in sensitizing MCF-7 cells to MDM2 inhibition. To test this, we inhibited DNA damage signaling in MCF-7 cells through inhibition of ATM/ATR-dependent checkpoints by caffeine (2) treatment ${ }^{24-26}$. The inhibition of ATM/ATR kinases in MCF-7 cells resulted in a decrease in the transcriptional activation by $\mathrm{p} 53$ in response to nutlin-3 exposure, as evidenced by induction of $\mathrm{p}^{1^{\text {cip } 1}}$ (Fig. 3b). The inhibition of 
a<smiles>COc1ccc(C2=NC(c3ccc(Cl)cc3)C(c3ccc(Cl)cc3)N2C(=O)N2CCNC(=O)C2)c(OC(C)C)c1</smiles>

b

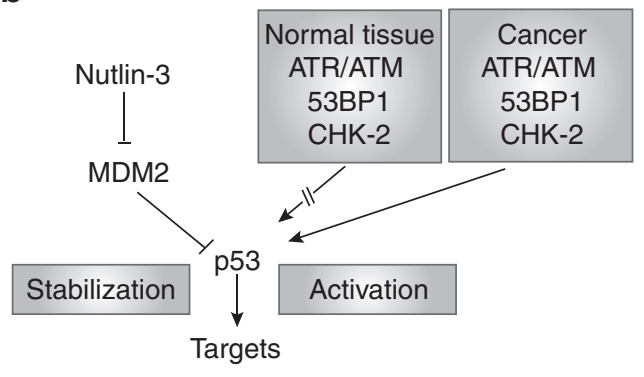

Figure 4 The cytotoxic effect of nutlin-3 in tumor cells is enhanced by intrinsic DNA damage signaling. (a) Chemical structure of nutlin-3. (b) Schematic representation of a putative model reflecting the intrinsic DNA damage signaling (involving 53BP1) present in tumor cells (but absent in normal cells) increasing the cytotoxicity associated with nutlin-3 treatment.
ATM/ATR kinases does not prevent p53 accumulation in response to nutlin-3 exposure, indicating that $\mathrm{p} 53$ stabilization and transactivation are separate events (Fig. 3b). To further test whether DNA damage signaling enhances the effects of nutlin-3, we treated normal BJ fibroblasts with increasing concentrations of nutlin- 3 in the absence or presence of a low level of DNA damage induced by ionizing radiation. A more robust activation of the $\mathrm{p} 53$ target gene $\mathrm{p} 21^{\mathrm{cip} 1}$ was seen in response to nutlin-3 treatment in the presence of DNA damage (Fig. $3 c$ ). From these results we conclude that inhibition of intrinsic DNA damage signaling by 53BP1 knockdown prevents full activation of p53 after stabilization in response to MDM2 inhibition.

Genomic instability is a well-recognized property of the vast majority of human cancers. This labels cancer tissues with a feature not seen in normal tissues, and it has therefore been suggested that genetic instability is an Achilles heel of tumor cells that could be exploited by future anticancer therapeutics ${ }^{27}$. Recent experiments have indicated that activation of DNA damage signaling (involving H2AX, 53BP1, ATR and ATM) occurs very early during tumorigenesis ${ }^{3,21}$. Our data indicate that this DNA damage signaling enhances the effects of the MDM2 inhibitor nutlin-3 (Fig. 4). The absence of such signals in normal cells in vivo may well explain their reduced sensitivity to nutlin-3 (ref. 2). The consequences of partial inactivation of MDM2 by small-molecule inhibitors resemble the phenotype seen in mice carrying a hypomorphic allele of MDM2. These mice are viable but show severe radiosensitivity ${ }^{28}$.

Our data suggest that MDM2 inhibitors would be most effective for tumors that have both wild-type p53 and activated DNA damage signaling, which is a substantial fraction of all human tumors. However, a combination of MDM2 inhibitors with DNA-damaging treatments may be harmful to normal cells and would cause undesired side effects in patients. In conclusion, the present study illustrates that shRNA barcode screens can be used to reveal cellular components that mediate drug cytotoxicity, resulting in a more complete understanding of drug action and enabling more rational decisions to be made in regard to drug application.

\section{METHODS}

Cell culture. MCF-7 breast cancer cells and BJ primary human fibroblasts, both expressing the ecotropic receptor, were cultured in DMEM supplemented with $10 \%$ fetal calf serum and penicillin and streptomycin. Cells were grown at $37^{\circ} \mathrm{C}$ in $5 \% \mathrm{CO}_{2}$. Phoenix cells were used to produce ecotropic retroviruses as described previously ${ }^{11}$. Nutlin-3 ((+/-)-4-[4,5-bis(4-chlorophenyl)-2-(2-isopropoxy4-methoxyphenyl)-4,5-dihydroimidazol-1-carbonyl]piperazin-2-one) was obtained from Cayman Chemical. Caffeine pretreatment of MCF-7 cells was performed by incubation with $2.5 \mathrm{mM}$ caffeine (Sigma-Aldrich) for $12 \mathrm{~h}$.

Barcode screen. MCF-7 cells were infected with retroviruses representing the complete Netherlands Cancer Institute (NKI) shRNA library described previously ${ }^{10}$. Infected cells were selected on puromycin $(2.0 \mu \mathrm{g} / \mathrm{ml})$ for $4 \mathrm{~d}$ and split into two populations. One population was left untreated, while the other population was exposed to $4 \mu \mathrm{M}$ nutlin-3. After $14 \mathrm{~d}$, genomic DNA was isolated with DNAzol (Invitrogen) and shRNA inserts were amplified from genomic DNA by PCR using the primers pRS-T7-fw, 5'-GGCCAGTGAATTGTAATACGACTCACTATAGGGAGGCGGCCCTTGAACCTCCTCGTTCGACC-3', containing a T7 RNA polymerase promoter sequence, and pRS8-rev, 5'-TAAAGCGCATGCTCCAGACT-3'. After purification (QIAquick PCR purification kit, Qiagen), PCR products were used for linear RNA amplification using the Megascript T7 kit (Ambion), and purified RNA probes (RNeasy, Qiagen) were labeled with cyanine-3 (Cy3) or cyanine-5 (Cy5) fluorescent groups using ULS (Kreatech) and purified over a KreaPure (Kreatech) spin column as described ${ }^{29}$. Labeled RNA probes from untreated and nutlin-3-treated cells were combined and hybridized to oligonucleotide arrays in $40 \mu \mathrm{l}$ of hybridization mixture ( $25 \%$ formamide, $5 \times$ SCC, $0.01 \%$ SDS, $25 \%$ Kreablock (Kreatech)). Samples were heated to $100^{\circ} \mathrm{C}$ for $5 \mathrm{~min}$ and applied to the array. Microarrays were hybridized for $18 \mathrm{~h}$ at $42^{\circ} \mathrm{C}$, washed and scanned using an Agilent microarray scanner. Quantification of the resulting fluorescent images was performed with Imagene 5.6 (BioDiscovery), local background was subtracted, and the data were normalized ${ }^{30}$ and ${ }^{2} \log$ transformed.

Plasmids. The shRNA sequences used to target $\mathrm{p} 53$ and $\mathrm{p} 53 \mathrm{BP} 1$ were as follows: $\mathrm{p} 53$, 5'-GACTCCAGTGGTAATCTAC-3'; 53BP1\#1, 5'-GATACTGCCTCATCACAGT$3^{\prime}$; 53BP1, 53BP1\#2 5'-GAACGAGGAGACGGTAATA- $3^{\prime}$. The shRNA sequences were cloned into pRETROSUPER as described previously ${ }^{10}$.

Cell-cycle analysis. MCF-7 cells infected with shRNA vectors against GFP (control), p53 and 53BP1 were treated for $48 \mathrm{~h}$ with nutlin-3 or left untreated. The cells were labeled with $10 \mu \mathrm{M}$ BrdU for 30 min before harvesting. Cells were fixed in $70 \%$ ethanol and permeabilized in $\mathrm{HCl}$-Triton solution $(2 \mathrm{M} \mathrm{HCl}$, $0.5 \%$ Triton $\mathrm{X}-100)$. The samples were neutralized in borate solution $(0.1 \mathrm{M}$ $\mathrm{Na}_{2} \mathrm{~B}_{4} \mathrm{O}_{7} \cdot 10 \mathrm{H}_{2} \mathrm{O}$ ) and labeled with antibody to BrdU (Dako; $1 \%$ BSA in PBS containing $0.5 \%$ Tween-20) in combination with FITC-conjugated secondary antibody (Dako; goat anti-mouse IGM-FITC). Samples were incubated with 10 $\mu \mathrm{g} \mathrm{ml}^{-1}$ propidium iodine and $250 \mu \mathrm{g} \mathrm{ml}^{-1} \mathrm{RNase}$. In each assay 10,000 cells were collected by FACScan (Becton Dickinson) and analyzed using the FCS Express program (De Novo Software).

Western blotting. Protein lysates were separated on SDS-PAGE gels, blotted onto nitrocellulose membranes and incubated with antibodies directed against MDM2 (2A10 and SMP14, Santa Cruz), 53BP1 (Novus Biologicals), p53 (DO-1), p21 (C19, Santa Cruz), CDK4 (C-22, Santa Cruz) and CDK2 (M2, Santa Cruz).

Immunofluorescence. Cells grown on coverslips were washed with PBS, fixed and permeabilized in $3 \%$ paraformaldehyde and $0.1 \%$ Triton X-100, and washed three times with PBS containing $0.05 \%$ saponin. Slides were blocked with $10 \%$ normal goat serum in PBS with $0.05 \%$ saponin. Cells were stained with antibodies directed against 53BP1 (1:100, Novus Biologicals) and against phosphorylated CHK-2 (p-T68-CHK2; 1:100, Cell Signaling Technologies); FITC-conjugated goat anti-rabbit antibodies were used as secondary antibodies. All antibodies were diluted in PBS containing 10\% normal goat serum and 0.05\% saponin.

Microarray data. Microarray datasets for the barcode experiments can found at the authors' supporting website (http://www.screeninc.nl). 


\section{LETTERS}

\section{ACKNOWLEDGMENTS}

We thank the Netherlands Cancer Institute microarray facility group for assistance; K. Berns, S. Nijman, H. Ovaa and members of the Bernards and Beijersbergen laboratories for help and discussions; L. Oomen for help with confocal microscopy; and R. Kortlever for critically reading the manuscript. This work was supported by grants from the Dutch Cancer Society (KWF) and the European Union integrated project INTACT.

\section{COMPETING FINANCIAL INTERESTS STATEMENT}

The authors declare that they have no competing financial interests.

Published online at http://www.nature.com/naturechemicalbiology

Reprints and permissions information is available online at http://npg.nature.com/ reprintsandpermissions/

1. Vassilev, L.T. et al. In vivo activation of the p53 pathway by small-molecule antagonists of MDM2. Science 303, 844-848 (2004).

2. DiTullio, R.A., Jr. et al. 53BP1 functions in an ATM-dependent checkpoint pathway that is constitutively activated in human cancer. Nat. Cell Biol. 4, 998-1002 (2002).

3. Gorgoulis, V.G. et al. Activation of the DNA damage checkpoint and genomic instability in human precancerous lesions. Nature 434, 907-913 (2005).

4. Lane, D.P. \& Fischer, P.M. Turning the key on p53. Nature 427, 789-790 (2004)

5. Vogelstein, B., Lane, D. \& Levine, A.J. Surfing the p53 network. Nature 408, 307 310 (2000).

6. Kubbutat, M.H., Jones, S.N. \& Vousden, K.H. Regulation of p53 stability by Mdm2. Nature 387, 299-303 (1997).

7. Kussie, P.H. et al. Structure of the MDM2 oncoprotein bound to the p53 tumor suppressor transactivation domain. Science 274, 948-953 (1996)

8. Zheng, X.S., Chan, T.F. \& Zhou, H.H. Genetic and genomic approaches to identify and study the targets of bioactive small molecules. Chem. Biol. 11, 609-618 (2004).

9. Harris, M.P. et al. Adenovirus-mediated p53 gene transfer inhibits growth of human tumor cells expressing mutant p53 protein. Cancer Gene Ther. 3, 121-130 (1996).

10. Berns, K. et al. A large-scale RNAi screen in human cells identifies new components of the p53 pathway. Nature 428, 431-437 (2004).

11. Brummelkamp, T.R. \& Bernards, R. New tools for functional mammalian cancer genetics. Nat. Rev. Cancer 3, 781-789 (2003).

12. Paddison, P.J. et al. A resource for large-scale RNA-interference-based screens in mammals. Nature 428, 427-431 (2004).

13. Westbrook, T.F. et al. A genetic screen for candidate tumor suppressors identifies REST. Cell 121, 837-848 (2005).
14. Janicke, R.U., Sprengart, M.L., Wati, M.R. \& Porter, A.G. Caspase-3 is required for DNA fragmentation and morphological changes associated with apoptosis. J. Biol. Chem. 273, 9357-9360 (1998).

15. Brummelkamp, T.R. et al. Functional identification of cancer-relevant genes through large-scale RNA interference screens in mammalian cells. Cold Spring Harb. Symp. Quant. Biol. 69, 439-445 (2004).

16. Moumen, A., Masterson, P., O'Connor, M.J. \& Jackson, S.P. hnRNP K: an HDM2 target and transcriptional coactivator of p53 in response to DNA damage. Ce// 123, 1065-1078 (2005)

17. Wu, M.X. Roles of the stress-induced gene IEX-1 in regulation of cell death and oncogenesis. Apoptosis 8, 11-18 (2003).

18. Wong, J., Li, P.X. \& Klamut, H.J. A novel p53 transcriptional repressor element (p53TRE) and the asymmetrical contribution of two p53 binding sites modulate the response of the placental transforming growth factor-beta promoter to p53. J. Biol. Chem. 277, 26699-26707 (2002).

19. Reinholz, M.M. et al. Differential gene expression of TGF beta inducible early gene (TIEG), Smad7, Smad2 and Bard1 in normal and malignant breast tissue. Breast Cancer Res. Treat. 86, 75-88 (2004).

20. Wang, B., Matsuoka, S., Carpenter, P.B. \& Elledge, S.J. 53BP1, a mediator of the DNA damage checkpoint. Science 298, 1435-1438 (2002).

21. Bartkova, J. et al. DNA damage response as a candidate anti-cancer barrier in early human tumorigenesis. Nature 434, 864-870 (2005).

22. Rappold, I., Iwabuchi, K., Date, T. \& Chen, J. Tumor suppressor p53 binding protein 1 (53BP1) is involved in DNA damage-signaling pathways. J. Cell Biol. 153 613-620 (2001).

23. Sedelnikova, O.A et al Senescing human cells and ageing mice accumulate DNA lesions with unrepairable double-strand breaks. Nat. Cell Biol. 6, 168-170 (2004).

24. Blasina, A., Price, B.D., Turenne, G.A. \& McGowan, C.H. Caffeine inhibits the checkpoint kinase ATM. Curr. Biol. 9, 1135-1138 (1999).

25. Zhou, B.B. et al. Caffeine abolishes the mammalian G(2)/M DNA damage checkpoin by inhibiting ataxia-telangiectasia-mutated kinase activity. J. Biol. Chem. 275 10342-10348 (2000).

26. Sarkaria, J.N. et al. Inhibition of ATM and ATR kinase activities by the radiosensitizing agent, caffeine. Cancer Res. 59, 4375-4382 (1999).

27. Lengauer, C., Kinzler, K.W. \& Vogelstein, B. Genetic instabilities in human cancers. Nature 396, 643-649 (1998).

28. Mendrysa, S.M. et al. mdm2 is critical for inhibition of p53 during lymphopoiesis and the response to ionizing irradiation. Mol. Cell. Biol. 23, 462-472 (2003).

29. Heetebrij, R.J. et al. Platinum(II)-based coordination compounds as nucleic acid labeling reagents: synthesis, reactivity, and applications in hybridization assays. ChemBioChem 4, 573-583 (2003).

30. Quackenbush, J. Microarray data normalization and transformation. Nat. Genet. 32 (Suppl.), 496-501 (2002) 